\title{
Modelling Droplet Deformation for Sneezing Events using the Eulerian-Lagrangian Particle Surface Approach
}

\author{
Filippo Gerbino*, Giovanni Tretola, Robert Morgan, Penny Atkins, Konstantina Vogiatzaki* \\ ${ }^{1}$ Advanced Engineering Centre, University of Brighton, Brighton, United Kingdom \\ *Corresponding author email: f.gerbino@brighton.ac.uk, k.vogiatzaki@brighton.ac.uk
}

\begin{abstract}
The Eulerian-Lagrangian approach is widely employed for spray simulations in various applications ranging from transportation systems to environmental flows. Traditional implementations of this technique are based on the assumption that the liquid droplets are spherical. This assumption is unphysical for several applications that droplets vary in shapes. The droplet shape and more specifically the droplet surface affects phenomena like evaporation and drag and consequently the droplet dynamics. Eulerian-Lagrangian simulations have been widely used in the modelling of infectious diseases. Experimental observations on respiratory droplets have shown that during a violent expiratory event, a sneeze, the exhaled saliva forms ligaments of various sizes. Since the shape of the droplets affects the evolution of the spray which in turn determines how far infectious droplets can travel, it is necessary to overcome the spherical assumption limitation in computational models. A novel spray model able to account for the droplet deformation is being developed and it is used in this paper to analyse the droplet deformation during a sneezing event. Results are compared to experimental observations. Such a new model can also be applied to spray injection within ICE or in jets in crossflow relevant to gas turbines.
\end{abstract}

\section{Keywords}

Eulerian-Lagrangian Simulations, Respiratory Droplets, Surface Density

\section{Introduction}

Sprays are of great interest in a wide range of applications. Their modelling has been based on two different approaches. The first one is the Eulerian-Lagrangian (EL) approach, in which the dispersed liquid phase is solved using a Lagrangian formulation, tracking individual droplet parcels and interpolating the gas-phase properties at the parcels locations. The second approach follows the Eulerian-Eulerian (EE) two-fluid methodology, treating different size classes of droplets as separate and inter-penetrating phases and solving conservation equations for each one of them. Though the EE framework can give more accurate results since it can provide predictions for any liquid structure in terms of shape, it requires a high computational cost. The EL offers a compromise in terms of computational cost and accuracy which justifies its widespread (and almost exclusive) use for large scale industrial internal combustion engines and gas turbine simulations, reproducing the global spray behaviour reasonably well. More recently the EL approach has also been used in biomedical applications. In particular, it has been used to investigate the transmission of respiratory infections by means of the mucosalivary droplets exhaled during violent expiratory events [1, 2], as well as by talking. The main limitation though of EL implementations is the spherical droplet assumption, which can lead to inaccuracies in the predictions of trans-critical and super-critical conditions where highly distorted droplets are present [3] or in set-ups such as cross flow jets where non spherical ligaments are present [4].

In this paper, a novel spray model able to account for the droplet deformation (based on the concept of the surface density approach[5]) has been developed within OpenFOAM. The model is capable of characterising the Lagrangian particles in terms of droplets geometry, in order to 
account for the surface and the elongation of the particle alongside their volume. Being able to model non-spherical droplets with the EL framework, means that we can predict the presence of ligaments and elongated structures, as well as flattened liquid structures, such as "bagshaped", "lens-shaped" and "spheroid" droplets in the flow. Eventually this can lead to more accurate characterisation of the the forces acting on the droplets and to improve several spray processes, such as droplets evaporation, drag force and droplet break-up.

This new spray model is applied here to study respiratory droplets exhaled during a sneeze. Respiratory droplets are linked to respiratory infections such as influenza, tuberculosis and severe acute respiratory syndrome (SARS), including the recent SARS-CoV-2 coronavirus. Experimental observations suggested that the exhaled droplets from various routes (speaking, coughing, sneezing) are not always spherical and thus simple EL frameworks used in previous studies might not be accurate. The formation of ligaments and sheets of saliva has been more pronounced during sneezing events[6]. Such structures undergo rapid fragmentation into smaller droplets after $100 \mathrm{~ms}$. Hence, the characterisation of the droplet shape is important as it provides information on the break-up and vaporisation of the particles of saliva exhaled during a sneeze. This, in turn can provide a better characterisation of the population of the saliva particles in terms of droplets size, determining the route of transmission of the infectious disease. To our knowledge this is the first numerical investigation of the droplet shape produced by sneeze.

\section{Numerical Modelling Lagrangian Particle Tracking method}

In the Lagrangian description of fluid flows, individual fluid particles are described by means of their properties (positions, velocities, etc.) as a function of time. Position vectors and velocity vectors are shown at one instant of time for each of these particles. As the particles move in the flow field, their positions and velocities change with time. The Lagrangian Particle Tracking (LPT) technique is commonly used in spray simulation. The spray is considered as a discrete phase comprising of a large number of parcels that are transported using Newton's second law. The basic equations of motion for the LPT method have been described in detail in our previous work [7].

\section{The Eulerian-Lagrangian Particle Surface Approach (ELPSA)}

The application of the Particle Surface Approach in the Lagrangian framework is based on the analogy of an oscillating and distorting droplet as a spring-mass-damper system. A parameter delta is calculated for every particle to describe the deviation from the spherical shape. The spherical deviation, $\delta$ (or "deformation factor"), helps to distinguish between ligament and lens shaped particles, affecting the break-up mechanisms. We assume that $\delta$ is negative for ligaments and positive for flattened droplets. Using the deformation factor we can then calculate the particle surface, which affects the vaporisation rate and the drag characteristics of the liquid structures.

The first assumption to model non-spherical droplets is to distinguish the geometrical characteristics of ligaments and flattened liquid structures. The assumptions made here is to consider the ligaments as "bullet"-shaped droplets and the flattened structures as lens-shaped droplets as shown in Fig.1.

As that same particle is subjected to different ambient conditions and/or forces, considering there is no vaporisation and/or breakup, its shape could change but its mass, and in turn its volume, remains constant. Being $V_{S p h}$ the volume of the particle, we can name $r_{s} p h$ the radius of the particle when it is spherical, which is provided by the numerical initialisation of the particle. The ligament and lens shaped particles are then characterised by two parameters, $r$ and $h$, 

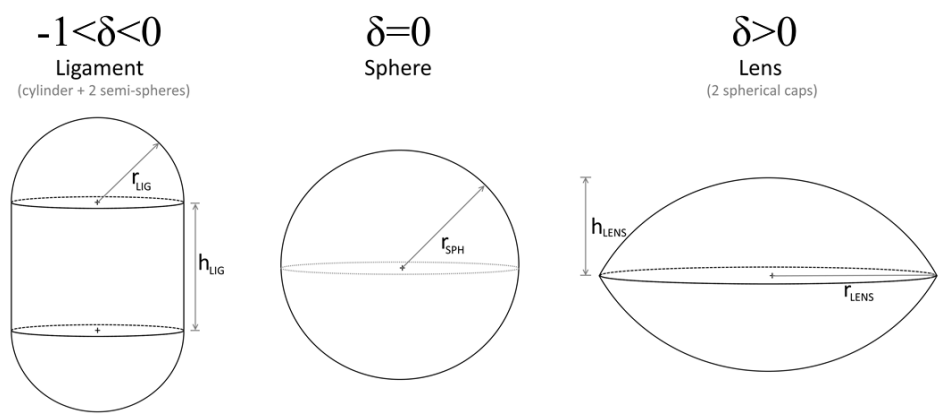

Figure 1. Geometrical shapes of the liquid droplets for the ligament, spherical and lens configuration.

that are both functions of $r_{s p h}$ and $\delta$. We can now describe the particle radius as:

$$
r=(1+\delta) r_{S p h}
$$

Based on the first assumption we made, we have:

$$
r_{\text {Ligament }} \leq r_{S p h} \leq r_{\text {Lens }}
$$

Notice that since $r$ is a positive real number $\delta$ must be $\delta>-1$. With the deformation factor we can now distinguish between ligament, lens and spherical shape, having:

$$
\delta= \begin{cases}-1<\delta \leq 0 & \Rightarrow r=r_{\text {Ligament }} \\ \delta=0 & \Rightarrow r=r_{\text {Sph }} \\ \delta \geq 0 & \Rightarrow r=r_{\text {Lens }}\end{cases}
$$

The height of the particle, $h$, is obtained from simple geometrical considerations. Two distinct equations are derived for $h$, one for each shape, which are function of $r_{s p h}$ and $\delta: h_{\text {Ligament }}=$ $h_{\text {Ligament }}\left(r_{\text {sph }}, \delta\right) a n d h_{\text {Lens }}=h_{\text {Lens }}\left(r_{\text {sph }}, \delta\right)$.

Hence we have:

$$
\delta= \begin{cases}-1<\delta \leq 0 & \Rightarrow h=h_{\text {Ligament }} \quad h \geq 0 \\ \delta \geq 0 & \Rightarrow h=h_{\text {Lens }} \quad 0 \leq h \leq r_{\text {Lens }}\end{cases}
$$

Two equation for the particle surface area are then obtained, one for the ligament and one for the disc-shaped particle:

$$
S_{\text {Ligament }}=S_{\text {Ligament }}\left(r, h_{\text {Ligament }}\right) \quad \text { and } \quad S_{\text {Lens }}=\left(r, h_{\text {Lens }}\right)
$$

Notice that if $\delta=0$, both the surface equations provides the same surface area, which is the surface of a spherical particle.

\section{Calculation of The Droplet Deformation Factor}

The $\delta$ is calculated within the LPT model using the Taylor-Analogy between an oscillating and distorting droplet and a spring mass system, similarly to the TAB model [8]. Here the surface tension and the viscosity play an important role on the droplets shape. The surface tension, $\sigma$, affects the resilience of the droplet; $\sigma$ opposes to deformation and forces the liquid surface to have the minimum area possible. The viscosity of the droplet, $\mu_{l}$, determines the "ductlity" of the liquid structures, that is the ability of the liquid particles to deform without breaking into smaller droplets; as $\mu_{l}$ increases the liquid particles can stretch and distort more extensively. The equation to solve is:

$$
\frac{d^{2} \delta}{d t^{2}}=\frac{c_{f}}{c_{b}}\left(\frac{\rho}{\rho_{l}}\right)\left(\frac{v_{\text {slip }}^{2}}{r_{S p h}^{2}}\right)-\frac{c_{k} \sigma}{\rho_{l} r_{S p h}^{2}} \delta-\frac{c_{d} \mu_{l}}{\rho_{l} r_{S p h}^{2}}\left(\frac{d \delta}{d t}\right)
$$


the terms on the RHS are respectively: the aerodynamic drag (external forces): $\frac{c_{f}}{c_{b}}\left(\frac{\rho}{\rho_{l}}\right)\left(\frac{v_{s l i p}^{2}}{r_{S p h}^{2}}\right)$; the surface tension effect (spring): $\frac{c_{k} \sigma}{\rho_{l} r_{S p h}^{2}} \delta$; the viscosity effect (damping): $\frac{c_{d} \mu_{l}}{\rho_{l} r_{S p h}^{2}}\left(\frac{d \delta}{d t}\right)$.

The model equation to be solved for the "distortion factor" is:

$$
\delta=W e+\exp \left(\frac{-\Delta t}{t_{d}}\right)\left[\delta_{0} \cos (\omega \cdot \Delta t)+\frac{\dot{\delta_{0}}}{\omega} \sin (\omega \cdot \Delta t)\right]
$$

where $W e=\frac{\rho_{l} u_{p}^{2} r_{S p h}}{\sigma}\left(\frac{1}{W e_{c r i t}}\right), t_{d}=\frac{2 \rho_{l} r_{S p h}^{2}}{c_{d} \mu_{l}}, \omega^{2}=\frac{c_{k} \sigma}{\rho_{l} r_{S p h}^{3}}-\frac{1}{t_{d}^{2}}, \delta_{0}=\delta-W e, \dot{\delta_{0}}=\dot{\delta}+\frac{\delta_{0}}{t_{d}}$; with $W e_{c r i t}=12, c_{d}=5$ and $c_{k}=8$ are model constant (these value are set as the TAB model default[8]).

A similar approach has been implemented by Nguyen et al. [9] to account for distorted droplets in the ECN spray A in trans critical conditions. The authors indicate that correctly accounting for the droplet shape leads to lower drag coefficients and enhanced evaporation of highly distorted droplets. In term of macroscopic quantities, there are improvements in liquid length prediction[9].

\section{Initialisation of the Sneezing}

Sneezing is a more violent expulsion compared to coughing. The velocity of the airflow exhaled by the sneeze is higher than that of breath and cough. Previous research $[6,10]$ has shown that sneezing events result in the generation of a sheet of fluid that expands and then breaks apart in long filaments that destabilise, and finally disperses as a spray of droplets. Another interesting aspect of sneezing is that the break up process depends to a large extent on the elasticity of the exhaled liquid. It has been suggested that the more elastic the fluid, or saliva, the longer the fluid travelled before breaking into droplets.

Not many studies have investigated the characterisation of sneezing events in terms of droplet size distribution and velocity. In Busco et al.[11] it is reported that the exhalation phase of sneeze can be separated into two parts. The first part consists of the ejection of aerosols and saliva droplets, while during the second part the remaining air in the lungs is expelled, for a total duration of approximately $0.5 \mathrm{~s}$. The saliva droplets injection lasts for $0.2 \mathrm{~s}$, as reported by Bourouiba et al. [12] and Scharfman et al.[13]. As for the case of cough, the peak of the airflow velocity is expected at $0.1 \mathrm{~s}$ [12]. In our study, the maximum and average airflow velocity for the sneeze have been selected according to Bahl et al.[14], being $v_{S, \max }=35 \mathrm{~m} / \mathrm{s}$ and $v_{S, a v g}=14 \mathrm{~m} / \mathrm{s}$. Though, as for cough, there are relevant discrepancies between different experimentalists for both the maximum and average airflow velocity. Accounting for the total sneeze duration and the maximum and the average value of the velocity, a time-varying inlet velocity is applied at the mouth patch for the sneeze airflow using a normal distribution to model it (see Fig.2a).

The droplets initial size distribution is based on experimental data from Han et al. [15] with some modifications. The initial distribution used here is shown in Fig. 2b. Notice that the experimental data by Han et al. [15] show a bi-modal distribution with a plateau in the droplets distribution within the range $3 x 10^{-4} \div 6 x 10^{-4} \mathrm{~m}$. Also in Han et al.[15] the authors do not report the presence of particles with diameters $<10 \mu \mathrm{m}$, while in our model they are accounted, according to what has been reported by other authors $[16,13]$. The mouth shape has been modelled based on the experimental observation by Busco et al.[11] on mouth opening during sneeze. From such measurements, it is shown that the shape is close to a rectangular surface with a length to height ratio of 2.18 [11]. For this reason, in this study the mouth patch is modelled as a rectangular surface with sides $19 \times 9 \mathrm{~mm}$. The total mass of saliva exhaled during 

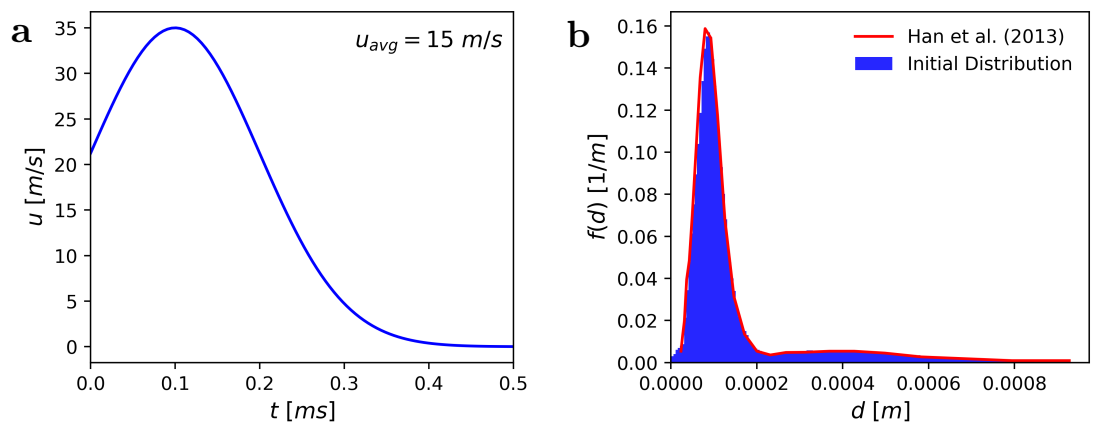

Figure 2. Velocity (a) and initial droplet size PDF (b) for a sneezing event. The initial PDF used here (blue area) is based on Han et al. [15] experimental findings (red line).

the sneeze was $7.7 \mathrm{mg}$.

\section{Results and discussion}

Results from the simulation are compared to the experimental data in terms of morphology and velocity. The experimental observations have been obtained by Scharfman et al. [6] using high-speed camera in the very first milliseconds of the sneeze, providing an insight into the fragmentation process of the saliva structures exhaled. High-speed camera have been also used by Bhal et al. [14], in a different set of experiments, to measure the velocity of the droplets by the Particle Tracking Velocimetry (PTV) method.

Figure 3 shows a comparison of the exhaled droplets of saliva obtained by the experiments of Scharfman et al. [6] to the Lagrangian particles obtained by the simulation. Notice that the spreading of the Lagrangian particles depends on the initialisation of airflow exhaled from the mouth, and it is not related to the implementation of the ELPSA model which explains some of the discrepancies in terms of sreading observed here.

We remind that the quantity $\delta$ represents the deformation of the particles, which is calculated implementing the ELPSA model. Note that blue represents the ligament-like droplets, while the red particle are the lens-shaped droplets. Referring to the simulations, $\delta=0$ for most of the particles, meaning they are spherical (see eq.3). The model predicts distorted droplets in the vicinity of the mouth. Although the experiments show many ligaments are formed, in particular at $0.021 \mathrm{~s}$, the ELPSA model exhibits only some of those features. The model predicts a high number of disc-shaped droplets, which represent the flattened liquid structures in that same region. On the other hand, the lower population of ligaments indicates the deficiency of the ELPSA model at the vicinity of mouth. Such an issue is linked both potentially to the current model formulation that needs further adjustments for extreme cases that only large ligaments are present (larger than the cell size) but also to other factors, such as the oral cavity shape and subject varying "initial" conditions that are not well documented in experiments. Coupling our model with a full Eulerian method (also based on the surface density approach) near the mouth might be beneficial but it will increase the computational cost. Despite this, the model can overall predict the temporal evolution of non-spherical droplets further downstream, in terms of their vaporisation and breakup, thus providing useful information on the sneeze evolution.

To get better insight for the area close to the mouth, in Fig.4 a close-up of the liquid structures provided by [6] is shown and compared to the particle deformation provided by the ELPSA model at $t=0.04 \mathrm{~s}$

For this area the ELPSA model is able to capture the flattened sheet of saliva which has been experimentally observed and which is represented by the high concentration of the red particles (lens-shaped droplets). On the other hand the model under-predicts the presence of ligaments (blue particles). Several ligaments have been experimentally observed in the first 

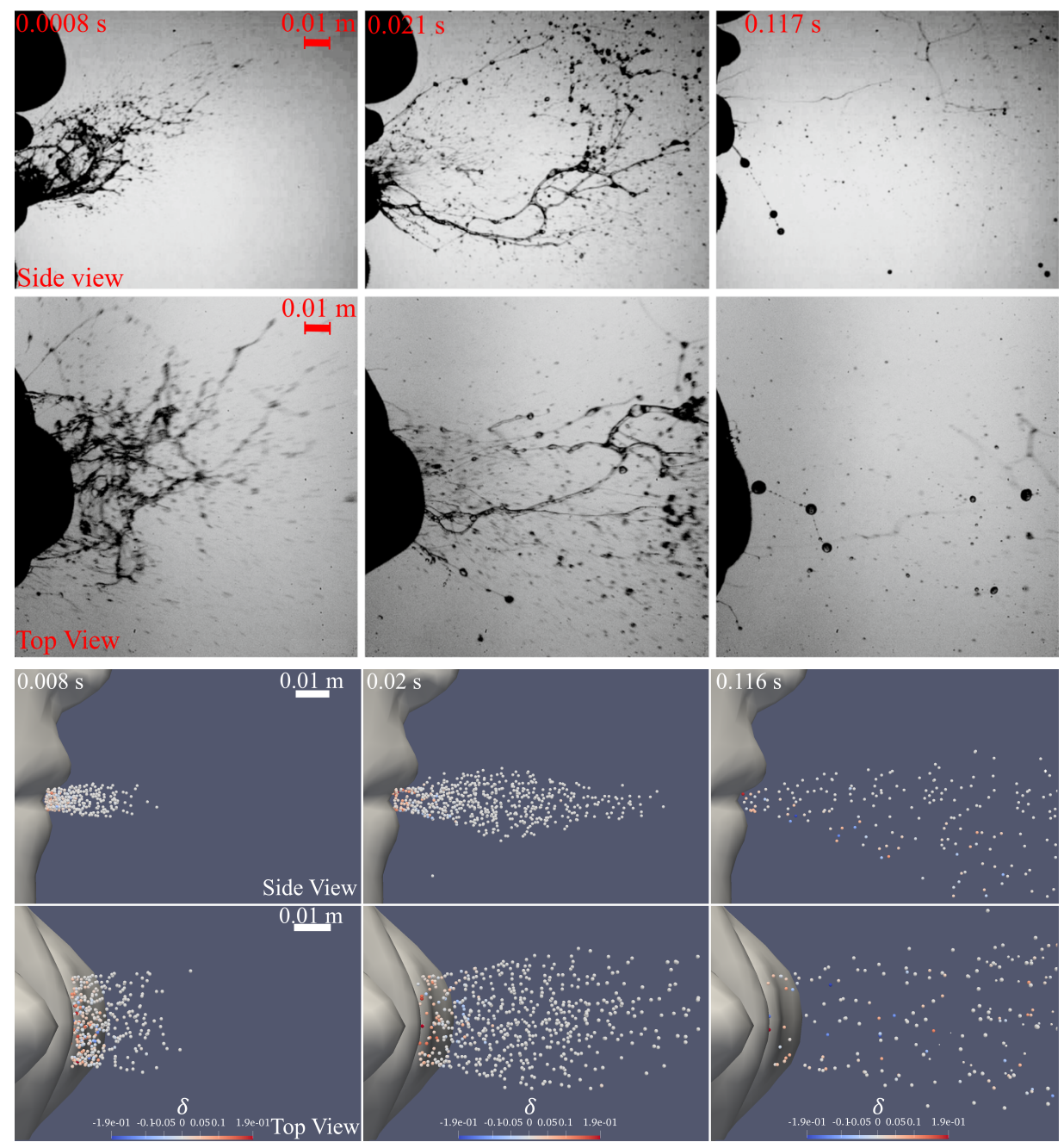

Figure 3. On top the experimental visualisation on the first stages of a sneeze adapted from [6]; on bottom the Lagrangian particle provided by the numerics.
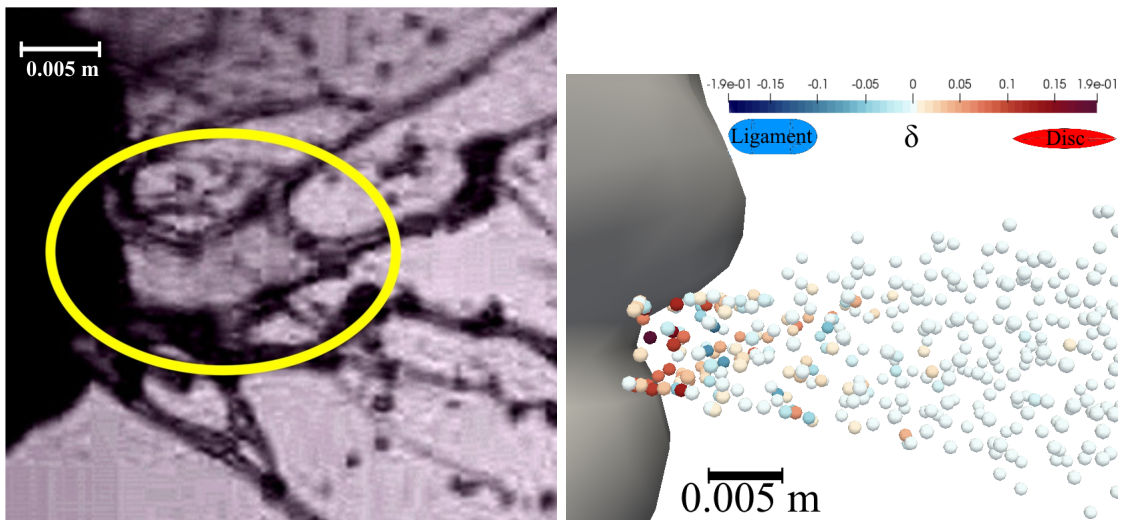

Figure 4. On the left hand side the liquid structures observed by Scharfaman et al. at $0.04 \mathrm{~s}$ [6]; on the right hand side the Lagrangian particle deformation obtained using the ELPSA model at $0.04 \mathrm{~s}$.

stages of the sneeze, from $0.008 \mathrm{~s}$ to 0.04 (see also Fig.3), though the numerical model tends to underestimate their number.

A further comparison to the experiments has been made in terms of droplet velocity. Such a parameter is important as it determines the droplet conditions, in particular its Weber number, also affecting the droplet deformation (see Eq.7). Figure 5 shows the comparison of the droplet velocity obtained using the PTV by Bhal et al. [14] and the velocity of the Lagrangian particles 
obtained from the simulations.
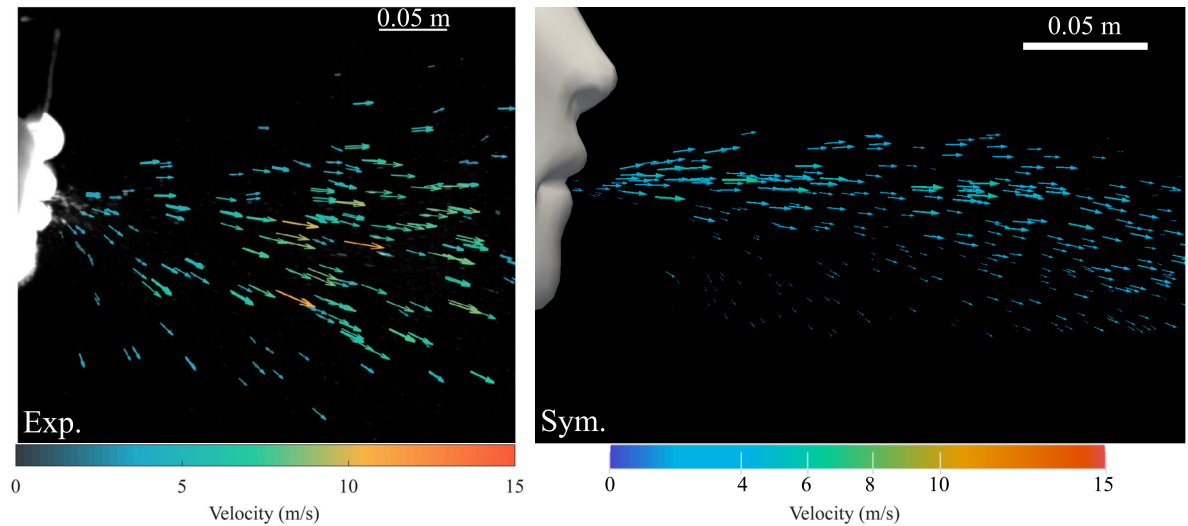

Figure 5. On the RHS: experimental observation of droplet velocity (adapted from [14]); on the LHS: Lagrangian particle velocity obtained from the simulations.

In general, simualations are able to quantitatively capture the droplet velocity. Bhal et al. [14] report that almost $80 \%$ of the droplets travel at velocities slower than $5 \mathrm{~m} / \mathrm{s}$, while less than $1 \%$ of the droplets expelled travel at velocities greater than $10 \mathrm{~m} / \mathrm{s}$. Though, it is important to notice that experimental observation of violent expiratory events from different authors show discrepancies for the values of several parameters, also due to the variability of participants characteristics. Such discrepancies also regards the droplet velocity [14]. A relevant difference between experiments and simulations is the region of maximum velocity of the droplets. While experiments shows that the particle with maximum velocity are located $0.15-0.2 \mathrm{~m}$ from the mouth, the simulations provides the maximum velocity closer to the mouth, 0.05-0.1 m downstream the mouth.

\section{Conclusions}

In this paper we presented and implemented in OpenFOAM a new model, the ELPSA model, within the Lagrangian methodology to capture the particle deformation during a sneeze. We investigated the details of the ejection of salivary fluid subject to an unsteady violent sneezeinduced turbulent flow. A direct comparison to the experimental observation shows that the model is able to capture some of the feature of the exhaled saliva structures, from a qualitative point of view. In particular the numerical model shows the presence of a disc-shaped droplets, which represent the thin film of fluid formed right at the mouth exit. Though, on the other hand the presence of ligaments is under-predicted by the ELPSA model which indicates that further modificaitons in the model are required. Being able to predict the presence of ligaments or flattened liquid structures is a big step forward with respect to the assumption of spherical Lagrangian particles. The shape of the particles affects several aspect of the droplets dynamic, such as the drag, the vaporisation and the break-up. The application of such a numerical tool to expiratory droplets is of paramount importance to investigate the route of transmission of respiratory infectious diseases. This is affected by the size of the droplets generated by sneeze and in turn by the atomisation due to the subsequent break-up. In fact, while the flattened particles should be affected by bag or catastrophic breakup (generating a cloud of very small particle, relatively to the parent droplet); ligaments are more likely to undergo vibrational breakup, (generating droplets whose size is roughly the ligament diameter). Hence it is possible to capture the generation of respiratory droplets and droplet nuclei. These aspects as well as the further model calibration will be performed in future investigations. 


\section{Acknowledgements}

The authors would like to thank the EPSRC support through the grant: EP/S001824/1 and the Doctoral Training Alliance (DTA) in Energy.

\section{References}

[1] Vuorinen, V., Aarnio, M., Alava, M., Alopaeus, V., Atanasova, N., Auvinen, M., Balasubramanian, N., Bordbar, H., Erästö, P., Grande, R., Hayward, N., Hellsten, A., Hostikka, S., Hokkanen, J., Kaario, O., Karvinen, A., Kivistö, I., Korhonen, M., Kosonen, R., Kuusela, J., Lestinen, S., Laurila, E., Nieminen, H. J., Peltonen, P., Pokki, J., Puisto, A., Råback, P., Salmenjoki, H., Sironen, T., and Osterberg, M., 2020. "Modelling aerosol transport and virus exposure with numerical simulations in relation to sars-cov-2 transmission by inhalation indoors". Safety Science, 130, p. 104866.

[2] Guo, L., Johnson, G. R., Hofmann, W., Wang, H., and Morawska, L., 2020. "Deposition of ambient ultrafine particles in the respiratory tract of children: A novel experimental method and its application". Journal of Aerosol Science, 139, p. 105465.

[3] Crua, C., Manin, J., and Pickett, L., 2017. "On the transcritical mixing of fuels at diesel engine conditions". Fuel, 208, pp. $535-548$.

[4] Mukundan, A., Tretola, G., Ménard, T., Herrmann, M., Navarro-Martinez, S., Vogiatzaki, K., César Brändle de Motta, J., and Berlemont, A., 2020. "Dns and les perspectives on atomization characteristics of turbulent liquid jet injection into gaseous crossflow". Accepted at Proceedings of the Combustion Institute.

[5] Ahmed, A., Tretola, G., Navarro-MartineZ, S., Vogiatzaki, K., Duret, B., Reveillon, J., and Demoulin, F.-X., 2020. "Atomization modeling using surface density and stochastic fields". Atomization and Sprays, 30(4), pp. 239-266.

[6] Scharfman, B. E., Techet, A. H., Bush, J. W. M., and Bourouiba, L., 2016. "Visualization of sneeze ejecta: steps of fluid fragmentation leading to respiratory droplets". Experiments in Fluids, 57(2), p. 24.

[7] Li, C., Crua, C., and Vogiatzaki, K., 2019. "Effect of the scale resolution on the two phase coupling characteristics of high speed evaporating sprays using les / eulerian-lagrangian methodologies". International Journal of Multiphase Flow, 120, p. 103060.

[8] O'Rourke, P. J., and Amsden, A. A., 1987. "The tab method for numerical calculation of spray droplet breakup". In SAE Technical Paper, SAE International.

[9] Nguyen, T. M., Rainer, N. D., and Pickett, L. M. "Modelling spray a with non-spherical droplets". ECN 6.9, ECN Web Meeting, 5 September 2019.

[10] Bourouiba, L., 2016. "A sneeze". New England Journal of Medicine, 375(8), p. e15.

[11] Busco, G., Yang, S. R., Seo, J., and Hassan, Y. A., 2020. "Sneezing and asymptomatic virus transmission". Physics of Fluids, 32, p. 073309.

[12] Bourouiba, L., Dehandschoewercker, E., and Bush, J. W. M., 2014. "Violent expiratory events: on coughing and sneezing". Journal of Fluid Mechanics, 745, p. 537-563.

[13] Scharfman, B., 2016. "Three dimensional imaging of multiphase flows". PhD thesis.

[14] Bahl, P., de Silva, C. M., Chughtai, A. A., Maclntyre, C. R., and Doolan, C., 2020. "An experimental framework to capture the flow dynamics of droplets expelled by a sneeze". Experiments in Fluids, 61(8), pp. 1432-1114.

[15] Han, Z. Y., Weng, W. G., and Huang, Q. Y., 2013. "Characterizations of particle size distribution of the droplets exhaled by sneeze". Journal of the Royal Society Interface, 10(88), p. 20130560.

[16] Pendar, M.-R., and Pascoa, J. C., 2020. "Numerical modelling of the distribution of virus carrying saliva droplets during sneeze and cough featured". Physics of Fluids, 32, p. 083305. 